\title{
Immunotherapy in surgically resectable non-small cell lung cancer
}

\author{
Dwight Owen ${ }^{1}$, Jamie E. Chaft ${ }^{2}$ \\ ${ }^{1}$ Division of Medical Oncology, Ohio State University Comprehensive Cancer Center, Columbus, OH, USA; ${ }^{2}$ Thoracic Oncology Service, \\ Department of Medicine, Memorial Sloan Kettering Cancer Center and Weill Cornell Medical College, New York, NY, USA \\ Contributions: (I) Conception and design: All authors; (II) Administrative support: All authors; (III) Provision of study materials or patients: All \\ authors; (IV) Collection and assembly of data: All authors; (V) Data analysis and interpretation: All authors; (VI) Manuscript writing: All authors; (VII) \\ Final approval of manuscript: All authors. \\ Correspondence to: Jamie E. Chaft, MD. 300 E 66th Street New York, NY 10065, USA. Email: chaftj@mskcc.org.
}

\begin{abstract}
Surgical resection is the mainstay of therapy for patients with resectable and operable early stage non-small cell lung cancer (NSCLC). Surgery alone yields an unacceptably high rate of lung cancer recurrence. The addition of chemotherapy to surgery as adjuvant or neoadjuvant treatment can improve survival rates by roughly $5 \%$ at 5 years. Recently, major advances in cancer immunotherapy have led to better outcomes for many patients with lung cancer. Monoclonal antibodies to programmed death 1 and its ligand are now approved for both first and second line treatment patients with metastatic lung cancer. In this review, we will outline the rationale and current research strategies investigating the role of immunotherapy in resectable NSCLC.
\end{abstract}

Keywords: Immunotherapy; surgery; adjuvant; neoadjuvant; non-small cell lung cancer (NSCLC)

Submitted Jul 11, 2017. Accepted for publication Dec 11, 2017.

doi: $10.21037 /$ jtd.2017.12.93

View this article at: http://dx.doi.org/10.21037/jtd.2017.12.93

\section{Background}

Lung cancer remains the leading cause of cancer-related death both in the United States and worldwide. Nonsmall cell lung cancer (NSCLC) is the most common histologic subtype (1). Patients who present with early stage disease are potentially cured with surgical intervention (2), however there is no cure for patients who develop metastatic disease after surgery. Even for patients who develop locally recurrent disease after surgery, the chance for cure is extremely low. For patients with early stage disease, the addition of systemic chemotherapy before or after surgery has been shown to improve 5 -year survival rates by roughly $5 \%$ across stages IB-IIIA, with a greater benefit in higher stages of disease (3-6). Treatment before surgery (neoadjuvant) allows for an assessment of treatment effect, including by major pathologic response (MPR) rate which has been associated with clinical outcomes for these patients and has been proposed as a surrogate endpoint for recurrence and survival $(7,8)$. Neoadjuvant clinical trials offer the opportunity to rapidly assess the efficacy of an intervention due to the availability of pre- and post-treatment specimens, and provide numerous translational research opportunities including the evaluation of potential biomarkers for response and for toxicity. Treatment with immunotherapy in the form of checkpoint inhibitors targeting programmed cell-death 1 (PD-1) and its ligand (PD-L1) has dramatically altered the management and outcomes of patients with NSCLC among many other tumors (9-12). A number of trials are currently evaluating whether anti-PD-1 and anti-PD-L1 agents can be incorporated into the care of patients with early stage NSCLC. This review will outline the data supporting the use of immunotherapy in the management of patients with early stage NSCLC, as well as detail the ongoing trials that may potentially change the treatment paradigm for these patients.

\section{Rationale for neoadjuvant and adjuvant treatment in early stage NSCLC}

Survival for early stage lung cancer remains low, with high 
numbers of patients succumbing to disease recurrence and 5-year survival rates dropping from $60 \%$ for stage IIA disease to $36 \%$ for stage IIIA disease, according to the new $8^{\text {th }}$ edition staging project of the International Association for the Study of Lung Cancer (13). This high rate of recurrence despite surgical resection of known disease has inspired investigators for decades to design studies to improve outcomes for these patients. One of the first studies to demonstrate a survival benefit for the addition of chemotherapy to surgical resection was a metaanalysis of 52 randomized clinical trials spanning 30 years, which suggested a $5 \%$ improvement in 5-year survival with the addition of chemotherapy to surgery [hazard ratio (HR) $0.87,13 \%$ reduction in the risk of death] (14). In the following years a number of prospective trials were performed to assess whether the addition of adjuvant chemotherapy to surgery could improve outcomes for patients with early stage lung cancer. The International Adjuvant Lung Cancer Trial (IALT) randomized 1,867 patients with stage I-III disease to surgery followed by either observation or one of several chemotherapy regimens, comprising a cisplatin backbone (at 80, 100, or $120 \mathrm{mg} / \mathrm{m}^{2}$ ) and either etoposide, vinorelbine, vinblastine, or vindesine. Patients who received chemotherapy had a $4.1 \% 5$-year survival advantage compared to those who were observed [HR for death $0.86,95 \%$ confidence interval (CI), 0.76-0.98] (15). This study was followed by the JBR.10 trial, a randomized phase 3 trial of 482 patients with stage IB or II NSCLC who received either observation or adjuvant chemotherapy with cisplatin $\left(50 \mathrm{mg} / \mathrm{m}^{2}\right.$ on day 1 and day 8$)$ and vinorelbine $\left(25 \mathrm{mg} / \mathrm{m}^{2}\right.$ weekly) after undergoing complete surgical resection (4). The updated overall survival (OS) advantage for patients treated with adjuvant chemotherapy at 5 years was $11 \%$ (16). With several additional prospective trials conducted around the same time using different chemotherapy regimens and including patients with varying stages of disease $(3,17)$, the LACE meta-analysis was conducted of the five largest cisplatin-based studies. With a median follow-up of 5.2 years, the LACE meta-analysis included 4,584 patients and demonstrated an absolute 5 -year survival benefit of $5.4 \%$ for all enrolled patients treated with adjuvant chemotherapy versus surgery alone (5). Based on these data, adjuvant cisplatin-based chemotherapy is now offered as a standard treatment option for patients who have completely resected NSCLC Stage IIA or greater are able to tolerate cytotoxic chemotherapy, and for select patients with large or high-risk Stage IB tumors.

\section{Timing of adjuvant and neoadjuvant therapy}

The timing of adjuvant treatment in clinical trials has generally been within 6 to 8 weeks of surgery $(3,4)$. However a large prospective registry trial of 3,354 patients with early stage NSCLC in Canada revealed that the median time to adjuvant chemotherapy was 8 weeks (range, 1-16 weeks), and although a significant amount of patients $(35 \%)$ were delayed up to 10 weeks, there was no association between delayed treatment and OS (18). More recently, a large retrospective study of 12,473 patients revealed that even when adjuvant chemotherapy was delayed up to 4 months, there was still a benefit over surgical resection only (19). The optimal timing of neoadjuvant and adjuvant immunotherapy treatment remains unclear. Many ongoing trials offer immunotherapy to patients after they receive standard of care adjuvant chemotherapy and/or radiation.

\section{Immunotherapy for early stage NSCLC}

The findings that treatment with immunotherapy targeting PD-1 and PD-L1 can improve outcomes compared to chemotherapy for select patients in the metastatic setting $(9,10,20,21)$ has led to a number of clinical trials incorporating immunotherapy in earlier stages of the disease, as discussed in detail below. In addition to the improved toxicity profile of immunotherapy compared to cytotoxic chemotherapy, there is evidence that immunerelated changes in the tumor microenvironment may be just as important in early stage NSCLC as in metastatic disease. A recent study evaluated the immune microenvironment of lung adenocarcinoma tumors compared to normal lung tissue and peripheral blood using paired single-cell analysis for 32 patients undergoing surgical resection for early stage disease (22). The authors reported data demonstrating that an immunosuppressive microenvironment had developed even in early stage adenocarcinoma, with increased regulatory $\mathrm{T}$ cells (Treg), reduced $\mathrm{T}$-effector/Treg ratio, and reduced natural Killer (NK) and dendritic cells in the tumor microenvironment compared to normal lung. These changes were present in patients with stage I disease as well as stage II and III disease. The finding of an immunetolerant environment even in the earliest stages of disease supports the investigation of immunotherapeutic strategies in this patient population. 
Experience with neoadjuvant and adjuvant immunotherapy in the treatment of patients with NSCLC has so far mostly been limited to vaccine trials and adoptive cell transfer studies. Adjuvant treatment with an immunotherapeutic targeting melanoma-associated antigen-A3 (MAGE-A3) was assessed in the MAGRIT study, one of the largest therapeutic trials ever performed in adjuvant NSCLC (23). MAGE-A3 is silent in nearly all normal human tissue but is expressed in up to half of NSCLC tumors, and based on promising results of a phase 2 trial (24), the phase 3 MAGRIT trial was undertaken which screened 13,849 patient tumor samples for MAGE-A3 expression, and eventually enrolled 2,312 patients to either MAGE-A3 immunotherapeutic [1,515] or placebo [757] following complete surgical resection of stage IB-IIIA NSCLC. Treatment with the MAGE-A3 immunotherapeutic did not improve disease free survival (DFS) or OS in the overall population of any of the pre-specified subgroup patient populations. Another vaccine evaluated in NSCLC was tecemotide (L-BLP25), a liposome peptide vaccine to mucin 1 (MUC1) glycoprotein which is over-expressed and glycosylated in up to two-thirds of NSCLC tumors, leading to increased cell growth and proliferation (25). A randomized phase 2 trial showed a trend towards improvement in OS when tecemotide was used as maintenance therapy after chemotherapy for patients with stage IIIB or IV NSCLC (26), and a subsequent phase 2 trial demonstrated promising efficacy when tecemotide was given after completion of chemoradiation for patients with stage III NSCLC (27). Based on these data, an international, randomized, phase 3, placebo-controlled, double-blind trial was conducted of 1,513 patients comparing tecemotide to placebo in patients with stage III NSCLC who had completed chemoradiation treatment (28). The study reported no significant difference in survival between the treatment arms, with a median OS for patients treated with tecemotide of 25.6 months compared to 22.3 months for placebo (HR, 0.88; 95\% CI, 0.75-1.03; $\mathrm{P}=0.123$ ).

Adoptive cell transfer therapy has been evaluated in a number of trials, including a phase 3 randomized trial of adjuvant chemo-immunotherapy with platinum doublet and autologous activated killer $\mathrm{T}$ cells and dendritic cells isolated from regional lymph nodes compared to chemotherapy alone in patients who had undergone resection for NSCLC (29). The trial included primarily patients with stage IB-IIIA disease, although notably the trial did allow patients after neoadjuvant chemotherapy and also included patients with stage IIIB or IV disease if malignant pleural effusion, micrometastases to mediastinal lymph nodes, or intrapulmonary metastases were discovered at time of surgery. The trial randomized 103 patients to either chemo-immunotherapy or chemotherapy alone and reported an improved in OS in the chemo-immunotherapy arm which reached statistical significance with a HR of 0.229 (95\% CI, 0.093-0.564, P=0.0013). Further research with these technologies is currently underway in the metastatic setting, including infusion of autologous tumor infiltrating lymphocytes (NCT02133196), as well as infusion of chimeric antigen receptor (CAR) $\mathrm{T}$ cells engineered against specific proteins including NY-ESO-1 (NCT02588612), or MUC-1 (NCT03179007).

More recently, multiple randomized, prospective clinical trials have demonstrated that treatment with PD-1 and PD-L1 inhibitors can lead to improved outcomes for select patients with metastatic NSCLC compared to chemotherapy (9-12). PD-L1 by immunohistochemistry has been developed concurrently as a potential biomarker for response to treatment with PD-1 and PD-L1 inhibitors, and the PD-1 inhibitor pembrolizumab is now approved for first line therapy of patients with NSCLC with high expression of PD-L1 (>50\% tumor proportion score) based on the results of KEYNOTE-24 (20). KEYNOTE-024 was a randomized, international, open-label, phase 3 trial which evaluated first-line treatment with pembrolizumab compared to chemotherapy for patients with PD-L1 TPS $>50 \%$. The study met its primary endpoint and demonstrated an improvement in PFS in patients treated with pembrolizumab compared to chemotherapy with a HR of 0.50 (95\% CI, $0.37-0.68 ; \mathrm{P}<0.001$ ). The study also showed an improvement in OS (HR, 0.60; 95\% CI, 0.41-0.89; $\mathrm{P}=0.005)$ and an improved response rate of $44.8 \%$ in patients treated with pembrolizumab compared to $27.8 \%$ in patients treated with chemotherapy. Based on this data, pembrolizumab is now approved for first-line treatment for patients with NSCLC with PD-L1 expression $>50 \%$. Given these outcomes and a better safety profile than platinum chemotherapy, a number of clinical trials are currently underway evaluating immunotherapy with checkpoint inhibitors in the neoadjuvant (Table 1) and adjuvant settings (Table 2).

An additional benefit of immunotherapy in early stage NSCLC is the possibility of generating a long-lasting antitumor immune response to micrometastatic disease, thus preventing disease relapse or recurrence. Preliminary data from the ongoing trial NCT02259621 evaluating the PD-1 inhibitor nivolumab for patients with early stage NSCLC 
Table 1 Selected trials of neoadjuvant immunotherapy for NSCLC

\begin{tabular}{|c|c|c|c|c|c|}
\hline Trial identifier & Phase & Sponsor & Stage & Intervention & Primary endpoint \\
\hline NCT03158129 & 2 & M.D. Anderson Cancer Center & I-IIIA & $\begin{array}{l}\text { Nivolumab with or without } \\
\text { ipilimumab }\end{array}$ & MPR \\
\hline NCT02818920 & 2 & Duke University Medical Center & IB-IIIA & $\begin{array}{l}\text { Pembrolizumab (neoadjuvant and } \\
\text { adjuvant) }\end{array}$ & Surgical feasibility rate \\
\hline NCT02927301 & 2 & LCMC-3, Genentech & IB-IIIA & Atezolizumab & MPR \\
\hline NCT02572843 & & $\begin{array}{l}\text { Swiss Group for Clinical Cancer } \\
\text { Research }\end{array}$ & IIIA (N2) & Durvalumab & Event-free survival (EFS) \\
\hline
\end{tabular}

NSCLC, non-small cell lung cancer; BMS, Bristol Meyer Squibb; LCMC, Lung Cancer Mutation Consortium; MPR, major pathologic response rate.

Table 2 Selected trials of adjuvant immunotherapy for NSCLC

\begin{tabular}{lcllll}
\hline Trial identifier & Phase & Sponsor & Stage & Intervention & Primary endpoint \\
\hline NCT02595944 & 3 & National Cancer Institute (NCI) & IB-IIIA & Nivolumab & DFS, OS \\
NCT02486718 & 3 & Hoffmann-La Roche & IB-IIIA & Atezolizumab & DFS \\
NCT02273375 & 3 & Canadian Cancers Trials Group & IB-IIIA & Durvalumab & DFS \\
NCT03130764 & 2 & Columbia University & IB-IIIA & Durvalumab and Tremelimumab & Induced T-cell response rate \\
NCT02504372 & 3 & EORTC, Merck & IB-IIIA & Pembrolizumab & DFS \\
NCT03053856 & 2 & Samsung Medical Center & IIIA (N2) & Pembrolizumab & DFS \\
\hline
\end{tabular}

NSCLC, non-small cell lung cancer; DFS, disease free survival. OS, overall survival. EORTC, European Organisation for Research and Treatment of Cancer.

were presented at the 2016 Congress of the European Society of Medical Oncology by Forde et al. (30). This pilot study showed that neoadjuvant immunotherapy was feasible without delaying curative surgery, and also demonstrated a $43 \%$ response rate as determined by MPR at the time of surgery (31). The structure of this neoadjuvant trial affords the collection of samples for translational research, including tumor mutational burden, TCR sequencing of T cells from the peripheral blood, tumor and regional lymph nodes, as well as multiplex immunohistochemistry and other correlates. A large neoadjuvant trial of the PD-L1 monoclonal antibody atezolizumab is currently underway at 15 academic centers participating in the Lung Cancer Mutation Consortium (NCT02927301). This is a singlearm, phase 2 trial which plans to accrue 180 patients with stage IB-IIIA NSCLC, which will assess the efficacy of two doses of neoadjuvant atezolizumab prior to curative intent surgery. The primary endpoint is MPR determined at the time of surgery, and will also evaluate adjuvant atezolizumab in an exploratory cohort of patients. The early experience has prompted launch of a Phase 3 study evaluating preoperative immunotherapy versus chemotherapy in earlystage, surgically resectable NSCLC (NCT02998528).

A number of trials of adjuvant PD-1/L1 agents are underway internationally in patients with completely resected NSCLC. For example, the Adjuvant Nivolumab in Resected Lung Cancers (ANVIL) trial is a randomized phase 3 comparing adjuvant nivolumab with observation after surgical resection and standard of care adjuvant chemotherapy and/or radiotherapy for patients with 
resected stage IB-IIIA NSCLC (NCT02595944). Atezolizumab, durvalumab, and pembrolizumab are each being evaluated in Phase 3 trials in the adjuvant setting. All four of these adjuvant efforts are evaluating a year of treatment. A single center exploratory adjuvant combination immunotherapy is planned. These are outlined in Table 2.

\section{Potential advantages of neoadjuvant treatment}

The advantages of neoadjuvant treatment prior to surgical resection have been considered mostly in the setting of cytotoxic chemotherapy, and include the reduction of tumor size to allow for a potentially less morbid resection (i.e., a lobectomy vs pneumonectomy), the ability of patients to tolerate therapy better before surgery compared to afterward, as well as the earlier eradication of nodal and micrometastatic disease (6). One of the only studies to directly compare neoadjuvant and adjuvant chemotherapy in NSCLC is the randomized, phase $3 \mathrm{NATCH}$ trial which demonstrated no difference in DFS between patients who received neoadjuvant or adjuvant cisplatin and paclitaxel chemotherapy compared to surgery alone, although the trial included a large number of stage 1 patients who do not typically benefit from these treatments (32). Of note, the trial did demonstrate that a higher proportion of patients in the neoadjuvant arm received all doses of planned chemotherapy compared to the patients in the adjuvant arm, supporting the suggestion that patients may tolerate treatment better in the neoadjuvant setting. The use of immunotherapy in the neoadjuvant setting may offer these advantages in addition to the theoretical development of a sustained anti-tumor immune response that can persist beyond surgery, thus offering protection from recurrent disease. This hypothesis was recently tested in a study utilizing an orthotopic murine triple-negative breast cancer model to compare neoadjuvant to adjuvant anti-PD-1 immunotherapy (33). The authors of this study reported that neoadjuvant immunotherapy followed by surgical resection in mice led to improved survival and reduced metastases compared to adjuvant treatment, and that this benefit was independent of metastatic disease burden. Promisingly, this study demonstrated that a brief duration of neoadjuvant treatment was capable of inducing long-term responses which seemed to be dependent on tumor-specific T-cells, and which persisted beyond surgical resection. In lung cancer patients, a prospective trial of neoadjuvant chemo-immunotherapy with cisplatin, docetaxel, and cetuximab demonstrated that a decrease in peripheral regulatory T-cells during treatment correlated with an increased response to in patients with Stage IB-IIIA NSCLC (34).

\section{Biomarkers in early stage NSCLC}

Thus far, efforts to identify biomarkers that may predict patients at higher risk of recurrence, or who would benefit most from the addition of adjuvant treatment have been disappointing. Biomarkers that had initially shown promise in early phase trials, including excision repair crosscomplementation group 1 (ERCC1) (35), NRF2, and MAGE-A3, have not yielded clinically useful results in larger prospective trials $(36,37)$.

In the prospective Tracking Non-Small Cell Lung Cancer Evolution through Therapy Consortium (TRACERx) trial, multi-region whole-exome sequencing was performed on resected tumor specimens of earlystage lung cancer patients, who were followed clinically throughout their disease course. In a report of the first 100 patients, pre-treatment chromosome instability and elevated copy-number heterogeneity were associated with increased risk of recurrence or death (38). A separate analysis from the TRACERx study revealed that levels of circulating tumor DNA (ctDNA) could predict disease relapse earlier than imaging with CT (median 70 days earlier, range 10 to 346 days), and post-surgical ctDNA may predict residual disease and therefore identify a subset of patients at higher risk of relapse (39).

Although PD-L1 by IHC is now a standard assessment for treatment of metastatic NSCLC (20), its role in early stage NSCLC is unclear. Although some variability between the different assays for PD-L1 certainly exists (40), it appears that the combination of PD-L1 and tumor mutational burden may be a better biomarker than either one alone, though even a combined score has an imperfect negative predictive value (41). This will need to be assessed prospectively in the clinical trials currently underway.

MPR has been proposed by several groups as a surrogate marker for recurrence and survival in patients with NSCLC after neoadjuvant treatment. MPR is assessed at the time of surgery, and is a defined as less than or equal to $10 \%$ viable tumor cells across different sections of a tumor at the time of surgery after neoadjuvant treatment. The use of pathologic complete response in breast cancer is well characterized and is an accepted surrogate marker for OS $(42,43)$; however pathologic complete responses in NSCLC are rare (44-46). MPR was assessed in a retrospective study 
of 192 patients with stage I-IV NSCLC who underwent surgical resection following neoadjuvant chemotherapy, and reported that patients with MPR $(\leq 10 \%$ viable tumor cells) had significantly improved survival compared to those who did not have a MPR at time of surgery (5-year OS $85 \%$ versus $40 \%, \mathrm{P}<0.0001$ ) (8). In a prospective trial of 50 patients with stage IB-IIIA NSCLC treated with neoadjuvant chemotherapy and bevacizumab, more patients who had achieved MPR at time of surgery were alive at three years compared to those who did not achieve MPR after neoadjuvant treatment ( $100 \%$ versus $49 \%, P=0.02$ adjusted for stage) (47). MPR is the primary endpoint of a number of ongoing trials of neoadjuvant immunotherapy (Table 1). These efforts will help define the validity this endpoint as a surrogate of survival as well as differences in pathologic response patterns to immunotherapy versus chemotherapy.

\section{Conclusions}

Immunotherapy with monoclonal antibodies to PD-1 and PD-L1 are now approved for treatment of patients with metastatic NSCLC, and have been shown in a number of randomized trials to lead to better outcomes for select patients compared to standard chemotherapy. Studies are currently underway to evaluate whether these benefits can be seen in patients with early stage disease, as well as evaluating the safest and most effective strategies to incorporate these treatments in the multi-disciplinary management of patients with resectable NSCLC. If these adjuvant trials do demonstrate an improvement in survival, large efforts will be needed to define the appropriate patients for these treatments and the optimal duration of therapy. Trials with additional immunotherapy agents, including combination immunotherapies, chemo-immunotherapy, and CAR-T cells, will build on our current understanding and add hopefully yield new therapies that lead to better outcomes for patients with early stage NSCLC.

\section{Acknowledgements}

Funding: This work was supported by the National Institutes of Health (grant numbers P30CA016058 and P30CA008748).

\section{Footnote}

Conflicts of Interest: Dr. Chaft serves as an advisor to
Genentech, AstraZeneca, Bristol Meyers Squibb, and Merck. Dr. Owen has no conflicts of interest to declare.

\section{References}

1. Siegel RL, Miller KD, Jemal A. Cancer Statistics, 2017. CA Cancer J Clin 2017;67:7-30.

2. Scott WJ, Howington J, Feigenberg S, et al. Treatment of non-small cell lung cancer stage I and stage II: ACCP evidence-based clinical practice guidelines (2nd edition). Chest 2007;132:234S-42S.

3. Douillard JY, Rosell R, De Lena M, et al. Adjuvant vinorelbine plus cisplatin versus observation in patients with completely resected stage IB-IIIA non-small-cell lung cancer (Adjuvant Navelbine International Trialist Association [ANITA]): a randomised controlled trial. Lancet Oncol 2006;7:719-27.

4. Winton T, Livingston R, Johnson D, et al. Vinorelbine plus cisplatin vs. observation in resected non-small-cell lung cancer. N Engl J Med 2005;352:2589-97.

5. Pignon JP, Tribodet H, Scagliotti GV, et al. Lung adjuvant cisplatin evaluation: a pooled analysis by the LACE Collaborative Group. J Clin Oncol 2008;26:3552-9.

6. NSCLC Meta-analysis Collaborative Group. Preoperative chemotherapy for non-small-cell lung cancer: a systematic review and meta-analysis of individual participant data. Lancet 2014;383:1561-71.

7. Hellmann MD, Chaft JE, William WN, Jr., et al. Pathological response after neoadjuvant chemotherapy in resectable non-small-cell lung cancers: proposal for the use of major pathological response as a surrogate endpoint. Lancet Oncol 2014;15:e42-50.

8. Pataer A, Kalhor N, Correa AM, et al. Histopathologic response criteria predict survival of patients with resected lung cancer after neoadjuvant chemotherapy. J Thorac Oncol 2012;7:825-32.

9. Borghaei H, Paz-Ares L, Horn L, et al. Nivolumab versus Docetaxel in Advanced Nonsquamous Non-Small-Cell Lung Cancer. N Engl J Med 2015;373:1627-39.

10. Brahmer J, Reckamp KL, Baas P, et al. Nivolumab versus Docetaxel in Advanced Squamous-Cell Non-Small-Cell Lung Cancer. N Engl J Med 2015;373:123-35.

11. Fehrenbacher L, Spira A, Ballinger M, et al. Atezolizumab versus docetaxel for patients with previously treated non-small-cell lung cancer (POPLAR): a multicentre, open-label, phase 2 randomised controlled trial. Lancet 2016;387:1837-46.

12. Garon EB, Rizvi NA, Hui R, et al. Pembrolizumab for 
the Treatment of Non-Small-Cell Lung Cancer. New England Journal of Medicine 2015;372:2018-28.

13. Goldstraw P, Chansky K, Crowley J, et al. The IASLC Lung Cancer Staging Project: Proposals for Revision of the TNM Stage Groupings in the Forthcoming (Eighth) Edition of the TNM Classification for Lung Cancer. J Thorac Oncol 2016;11:39-51.

14. Chemotherapy in non-small cell lung cancer: a metaanalysis using updated data on individual patients from 52 randomised clinical trials. BMJ 1995;311:899-909.

15. Arriagada R, Bergman B, Dunant A, et al. Cisplatinbased adjuvant chemotherapy in patients with completely resected non-small-cell lung cancer. $\mathrm{N}$ Engl J Med 2004;350:351-60.

16. Butts CA, Ding K, Seymour L, et al. Randomized phase III trial of vinorelbine plus cisplatin compared with observation in completely resected stage IB and II nonsmall-cell lung cancer: updated survival analysis of JBR-10. J Clin Oncol 2010;28:29-34.

17. Scagliotti GV, Fossati R, Torri V, et al. Randomized study of adjuvant chemotherapy for completely resected stage I, II, or IIIA non-small-cell Lung cancer. J Natl Cancer Inst 2003;95:1453-61.

18. Booth CM, Shepherd FA, Peng Y, et al. Time to adjuvant chemotherapy and survival in non-small cell lung cancer: a population-based study. Cancer 2013;119:1243-50.

19. Salazar MC, Rosen JE, Wang Z, et al. Association of Delayed Adjuvant Chemotherapy With Survival After Lung Cancer Surgery. JAMA Oncol 2017;3:610-9.

20. Reck M, Rodriguez-Abreu D, Robinson AG, et al. Pembrolizumab versus Chemotherapy for PD-L1Positive Non-Small-Cell Lung Cancer. N Engl J Med 2016;375:1823-33.

21. Rittmeyer A, Barlesi F, Waterkamp D, et al. Atezolizumab versus docetaxel in patients with previously treated non-small-cell lung cancer (OAK): a phase 3, openlabel, multicentre randomised controlled trial. Lancet 2017;389:255-65.

22. Lavin Y, Kobayashi S, Leader A, et al. Innate Immune Landscape in Early Lung Adenocarcinoma by Paired Single-Cell Analyses. Cell 2017;169:750-65. e17.

23. Vansteenkiste JF, Cho BC, Vanakesa T, et al. Efficacy of the MAGE-A3 cancer immunotherapeutic as adjuvant therapy in patients with resected MAGE-A3-positive nonsmall-cell lung cancer (MAGRIT): a randomised, doubleblind, placebo-controlled, phase 3 trial. Lancet Oncol 2016;17:822-35.

24. Vansteenkiste J, Zielinski M, Linder A, et al. Adjuvant
MAGE-A3 immunotherapy in resected non-small-cell lung cancer: phase II randomized study results. J Clin Oncol 2013;31:2396-403.

25. Raina D, Kosugi M, Ahmad R, et al. Dependence on the MUC1-C oncoprotein in non-small cell lung cancer cells. Mol Cancer Ther 2011;10:806-16.

26. Butts C, Murray N, Maksymiuk A, et al. Randomized phase IIB trial of BLP25 liposome vaccine in stage IIIB and IV non-small-cell lung cancer. J Clin Oncol 2005;23:6674-81.

27. Butts C, Murray RN, Smith CJ, et al. A multicenter openlabel study to assess the safety of a new formulation of BLP25 liposome vaccine in patients with unresectable stage III non-small-cell lung cancer. Clin Lung Cancer 2010;11:391-5.

28. Butts C, Socinski MA, Mitchell PL, et al. Tecemotide (L-BLP25) versus placebo after chemoradiotherapy for stage III non-small-cell lung cancer (START): a randomised, double-blind, phase 3 trial. Lancet Oncol 2014;15:59-68.

29. Kimura H, Matsui Y, Ishikawa A, et al. Randomized controlled phase III trial of adjuvant chemoimmunotherapy with activated killer $\mathrm{T}$ cells and dendritic cells in patients with resected primary lung cancer. Cancer Immunol Immunother 2015;64:51-9.

30. Forde PM, Smith KN, Chaft JE, et al. Neoadjuvant antiPD1, nivolumab, in early stage resectable non-small cell lung cancer. 2016 ESMO Congress. Abstract LBA41_PR. Presented October 7, 2016.

31. Chaft JE, Forde PM, Smith KN, et al. Neoadjuvant nivolumab in early-stage, resectable non-small cell lung cancers. J Clin Oncol 2017;35:abstr 8508.

32. Felip E, Rosell R, Maestre JA, et al. Preoperative chemotherapy plus surgery versus surgery plus adjuvant chemotherapy versus surgery alone in early-stage nonsmall-cell lung cancer. J Clin Oncol 2010;28:3138-45.

33. Liu J, Blake SJ, Yong MC, et al. Improved Efficacy of Neoadjuvant Compared to Adjuvant Immunotherapy to Eradicate Metastatic Disease. Cancer Discov 2016;6:1382-99.

34. Pircher A, Gamerith G, Amann A, et al. Neoadjuvant chemo-immunotherapy modifies CD4(+)CD25(+) regulatory $\mathrm{T}$ cells (Treg) in non-small cell lung cancer (NSCLC) patients. Lung Cancer 2014;85:81-7.

35. Olaussen KA, Dunant A, Fouret P, et al. DNA repair by ERCC1 in non-small-cell lung cancer and cisplatin-based adjuvant chemotherapy. N Engl J Med 2006;355:983-91.

36. Friboulet L, Olaussen KA, Pignon JP, et al. ERCC1 isoform expression and DNA repair in non-small-cell lung 
cancer. N Engl J Med 2013;368:1101-10.

37. Wislez M, Barlesi F, Besse B, et al. Customized adjuvant phase II trial in patients with non-small-cell lung cancer: IFCT-0801 TASTE. J Clin Oncol 2014;32:1256-61.

38. Jamal-Hanjani M, Wilson GA, McGranahan N, et al. Tracking the Evolution of Non-Small-Cell Lung Cancer. N Engl J Med 2017;376:2109-21.

39. Abbosh C, Birkbak NJ, Wilson GA, et al. Phylogenetic ctDNA analysis depicts early stage lung cancer evolution. Nature 2017;545:446-51.

40. Hirsch FR, McElhinny A, Stanforth D, et al. PDL1 Immunohistochemistry Assays for Lung Cancer: Results from Phase 1 of the Blueprint PD-L1 IHC Assay Comparison Project. J Thorac Oncol 2017;12:208-22.

41. Carbone DP, Reck M, Paz-Ares L, et al. First-Line Nivolumab in Stage IV or Recurrent Non-Small-Cell Lung Cancer. N Engl J Med 2017;376:2415-26.

42. Fisher B, Bryant J, Wolmark N, et al. Effect of preoperative chemotherapy on the outcome of women with operable breast cancer. J Clin Oncol 1998;16:2672-85.

43. Guarneri V, Broglio K, Kau SW, et al. Prognostic value of

Cite this article as: Owen D, Chaft JE. Immunotherapy in surgically resectable non-small cell lung cancer. J Thorac Dis 2018;10(Suppl 3):S404-S411. doi: 10.21037/jtd.2017.12.93 pathologic complete response after primary chemotherapy in relation to hormone receptor status and other factors. J Clin Oncol 2006;24:1037-44.

44. Pisters KM, Kris MG, Gralla RJ, et al. Pathologic complete response in advanced non-small-cell lung cancer following preoperative chemotherapy: implications for the design of future non-small-cell lung cancer combined modality trials. J Clin Oncol 1993;11:1757-62.

45. Rosell R, Gomez-Codina J, Camps C, et al. A randomized trial comparing preoperative chemotherapy plus surgery with surgery alone in patients with non-small-cell lung cancer. N Engl J Med 1994;330:153-8.

46. Roth JA, Fossella F, Komaki R, et al. A randomized trial comparing perioperative chemotherapy and surgery with surgery alone in resectable stage IIIA non-small-cell lung cancer. J Natl Cancer Inst 1994;86:673-80.

47. Chaft JE, Rusch V, Ginsberg MS, et al. Phase II trial of neoadjuvant bevacizumab plus chemotherapy and adjuvant bevacizumab in patients with resectable nonsquamous nonsmall-cell lung cancers. J Thorac Oncol 2013;8:1084-90. 